
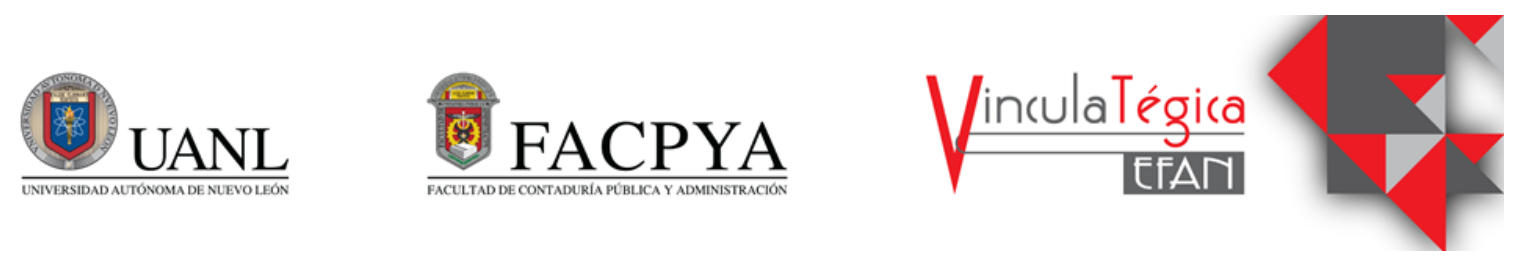

\title{
La innovación en los micronegocios en tiempo de pandemia. El caso de la plataforma Wabi
}

\author{
Ana María García García ${ }^{1}$, Mauricio Vargas Piñón ${ }^{2}$ y Minerva Hernández Aguilar ${ }^{3}$ \\ ${ }^{1}$ Universidad Intercultural del Estado de Puebla, procesadora.alimentos@uiep.edu.mx, 2227513662. \\ ${ }^{2}$ Universidad del Valle de Puebla, mauricio.vargas@uvp.edu.mx, 2223132916. \\ ${ }^{3}$ Universidad Intercultural del Estado de Puebla, s.academica@uiep.edu.mx,2225635038.
}

\begin{abstract}
Información del artículo revisado por pares
Fecha de aceptación: junio-2021

Fecha de publicación en línea: diciembre-2021

DOI: https://doi.org/10.29105/vtga7.2-65
\end{abstract}

\section{Resumen}

La situación de distanciamiento social derivada de la pandemia por el COVID-19, donde fue necesario que las personas se mantuvieran aisladas dentro de sus hogares, afectó con la reducción de ventas de los micronegocios conocidos como tienditas de la esquina. Este grupo de negocios dependían para sus ventas del tránsito de personas, por lo que bajo las condiciones de restricción de movilidad su permanencia se vio comprometida. Derivado del poco conocimiento que este tipo de negocios tenían sobre el comercio electrónico, no se encontraban en condiciones de poder migrar o complementar su actividad comercial con el apoyo de las TICs. El surgimiento de plataformas como Wabi, resultó en una alternativa viable para mantenerse vigentes en el mercado. El objetivo del presente artículo es reportar los resultados del pilotaje del instrumento a emplear en entrevistas estructuradas y así determinar las razones y beneficios percibidos por los micronegocios para ingresar al comercio electrónico, derivado de la situación provocada por la pandemia por COVID-19. Para ello, se hicieron entrevistas estructuradas a tendajones, misceláneas y tienditas de la esquina, para sondear su experiencia en Wabi.
Abstract

The situation of social distancing derived from the COVID-19 pandemic, where it was necessary for people to remain isolated within their homes, affected the reduction in sales of microbusinesses known as corner stores. This group of businesses depended for their sales on the transit of people, so under the conditions of restricted mobility their permanence was compromised. Derived from the little knowledge that this type of business had about electronic commerce, they were not in a position to migrate or complement their commercial activity with the support of ICTs. The emergence of platforms such as Wabi, resulted in a viable alternative to remain current in the market.

The objective of this article is to report the results of the piloting of the instrument to be used in structured interviews and thus determine the reasons and benefits perceived by microbusinesses to enter electronic commerce, derived from the situation caused by the COVID-19 pandemic. To do this, structured interviews were held with tendajones, misceláneas and corner stores, to probe their experience in Wabi. The results showed that adjustments should be made to the instrument in the questions related to the variables, driving benefits and problems, since at various times they are identified as closely related elements. However, after piloting the test, the rest 
Los resultados demostraron que deben hacerse adecuaciones al instrumento en las preguntas relacionadas con las variables, beneficios impulsores y problemas, ya que en varios momentos se llegan a identificar como elementos estrechamente relacionados. Sin embargo, después del pilotaje de la prueba, se considera que el resto de las preguntas del instrumento es pertinente y permite medir las variables de manera adecuada.

Palabras clave: Wabi, tienda de la esquina, comercio electrónico, impulsores, problemas

\section{INTRODUCCIÓN}

La existencia de la pandemia durante el 2020 y lo que va del 2021, ha puesto a prueba a muchas empresas, no solamente a las grandes, sino en especial han sido afectado los micronegocios, como es el caso de las misceláneas, minisúper y las llamadas tiendas de la esquina que, aunque no se vieron obligadas a suspender su actividad sí vieron reducidos sus ingresos derivados que el resto de la sociedad tuvieron que atender al llamado de aislamiento social durante el inicio de la pandemia.

Derivado de la situación anterior, la gran mayoría de este tipo de negocios no estaba preparado para hacer frente a una ausencia de los clientes o a una reducción de ventas de tal magnitud que comprometió la supervivencia de estos negocios o al menos representó la disminución de la planta de empleados. Como una respuesta defensiva estos micronegocios tuvieron que hacer adecuaciones a su modelo de negocio, redefiniendo la manera en que se relacionaban con sus clientes, los canales de comunicación y los socios claves. Por ello tuvieron que identificar alternativas que le permitieron seguir en contacto con sus clientes para continuar vendiendo.

Una posibilidad que surgió fue el of the questions in the instrument are considered pertinent and allow the variables to be adequately measured.

Keywords: Wabi, corner store, e-commerce, drivers, problems

JEL: E-commerce (L81), Sector studies: food and beverages (L66), Information technology management (M15).

comercio electrónico, sin embargo, estos negocios no tenían las capacidades y competencias necesarias que otras empresas de mayor tamaño tenían a su alcance. Esto les colocó en una grave encrucijada.

Afortunadamente, otros emprendedores desarrollaron alternativas para poder ayudar a estos negocios a ingresar al comercio electrónico a través del desarrollo de apps o plataformas que proporcionaran la infraestructura de comunicación con el cliente y la logística para continuar vendiendo. Un ejemplo de estas apps es Wabi, que se ha especializado en las conocidas "tienditas de la esquina".

El objetivo del presente artículo presentar los resultados del pilotaje del instrumento empleado para identificar las razones y beneficios percibidos por los micronegocios para integrarse al comercio electrónico a través de plataformas o apps, derivado de la situación económica generada por la pandemia del COVID-19 en la Ciudad de Puebla, México. 
Figura 1. Unidades económicas de comercio al por menor

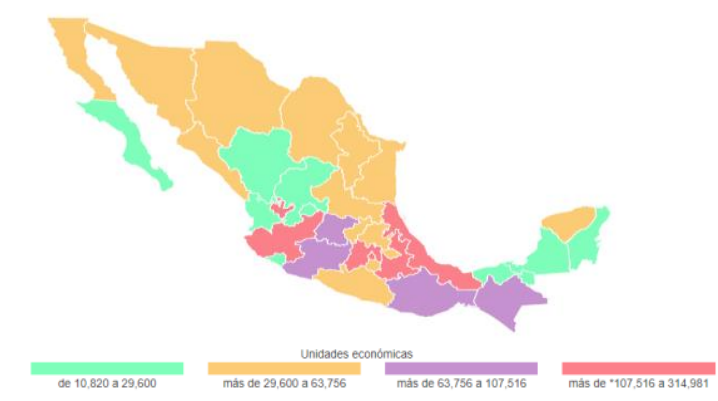

Fuente: INEGI, 2020.

\section{MARCO TEÓRICO}

Los micronegocios son una de las principales fuentes de empleo en el país, según el INEGI (2020a), En México se reportan más 6 millones de unidades económicas (UE), de las cuales más de 2 millones corresponden a las empresas de comercio al por menor, las que en total emplean a 5,899,054 personas $(16.37 \%$ del total nacional) (Figura 1).

En comparación con 2014, las tiendas de conveniencia han crecido un $46 \%$ más para 2019 (INEGI, 2020b). En el caso de Puebla (INEGI, 2020a), las UE reportadas en el Censo Económico del 2019, asciende a 134, 900 y emplean al $5.21 \%$ de las personas empleadas en el sector de comercio al por menor a nivel nacional. Dentro de estos negocios el $2.55 \%$ corresponde a las tiendas de abarrotes, ultramarinos, misceláneas, excluyendo las UE grandes.

En otro sentido, Martínez (2013) define como una microempresa a "la unidad de negocios donde se aprende, distribuye y desarrollan saberes productivos" (p. 176), asimismo indica que sus principales características son:

1. Tamaño reducido, tanto en número de empleados como en recursos financieros y materiales.

2. Condición de informalidad, ya sea por a) el tipo de mercado que atienden (marginales o subsidiarios y complementarios); b) albergar a sectores de la población marginados del empleo formal; y c) porque sus actividades quedan parcial o totalmente fuera de marcos institucionales.

3. Precariedad en ingresos, reducida relación capital-mano de obra, transacciones informales y recursos tecnológicos escasos u obsoletos.

4. Una carga persistente de racionalidad afectiva relacionada con la familia, amistades, entorno social y geográficos, inmediatos. Generando contradicciones entre fines, medio y productividad.

De las características mencionadas anteriormente resalta el hecho de que este tipo de microempresas, donde se integran las tiendas de abarrotes, gestionan el negocio en medio de grandes carencias, en especial de las relacionadas con el acceso y manejo de tecnologías como las TICs, mismas que son de gran influencia para el ingreso y permanencia dentro del comercio electrónico derivada de las condiciones de la pandemia.

Fontalvo (2013) menciona que el comercio electrónico tiene como ventajas:

1. Desaparecer barreras de localización geográfica que limitan su crecimiento debido a su actual localización,

2. Permite la comercialización de productos todo el año,

3. A través del uso de las TICs se facilita la intercomunicación con los clientes,

4. Se reduce el costo de publicidad,

5. No se limita a una sola población, sino que puede ampliar su mercado.

6. Las transacciones se hacen de manera ágil y oportuna,

7. Al evitar desplazamientos físicos se permite mayor movilidad y satisfacción del cliente,

8. Al reducir la cadena de intermediación se generan más ganancias para el cliente y la empresa.

9. Gracias al internet, las empresas pequeñas pueden ingresar a un mercado globalizado en igualdad de condiciones,

10. Las transacciones electrónicas aseguran previamente el pago del producto,

11. El cliente puede revisar información en el momento que lo desee y de manera inmediata.

12. Permite informar a clientes y 
proveedores sobre promociones o necesidades del mercado,

13. Ayuda a preservar el medio ambiente y permite que el negocio alcance la sostenibilidad a lo largo de los años.

Estas empresas usan tecnología que en muchas de las ocasiones, ni siquiera le permite tener acceso a la inclusión financiera ya que muchas de ellas siguen haciendo sus transacciones con clientes y proveedores a través del uso de efectivo. Por ejemplo, INEGI (2014) reporta que solo el $3.3 \%$ de las tiendas de abarrotes usaban equipo de cómputo y $18 \%$, internet. A diferencia de éstas, las tiendas de conveniencia, $62.5 \%$ de ellas contaban con computadora y 94.1, con acceso a internet, el cual usaban para búsqueda de información, gestión de negocio y operaciones bancarias y financieras. Adicionalmente, si se considera la cuestión de género, en el Censo Económico de 2019, el INEGI reporta una diferencia de $10 \%$ de la UE con equipo de cómputo disponible, de propietarios hombres en relación con UE de propietarias mujeres. Algo semejante pasa con el acceso a internet, donde un $8.8 \%$ de las UE de propietarios hombres cuentan con internet en comparación con las UE de mujeres (Figura 2).

Figura 2. TICs en UE de comercios al por menor en México

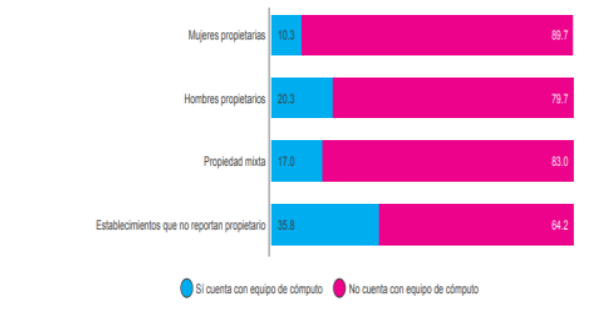

Fuente: INEGI, 2019

Si bien es cierto que estos micronegocios ya empiezan a apoyarse en la recepción de pago con tarjeta de débito, a través de los dispositivos que no le requieren integrarse a un sistema de banca y que no les obliga a tener una venta mínima mensual o a pagar la renta del dispositivo, también es cierto que muchos de ellos prefieren seguir recibido el pago en efectivo ya que ello les permite hacer la disposición inmediata para cubrir las necesidades del negocio y las personales.

\subsection{Wabi}

Esta aplicación nace en 2019 con el propósito de unir a comercios (tiendas de abarrotes, tendajones y misceláneas, es decir, "la tiendita de la esquina"), con personas que prefieren no salir de su casa. Es un emprendimiento de Coca-Cola, operado por la incubadora YopDev y se encuentra disponible en varios países, pero en el caso de México está operando en CdMx, Guadalajara, Puebla, Colima, Pachuca y Tijuana. Wabi se basa en la economía colaborativa, con el propósito de apoyar a la continuidad de micronegocios y que mantengan su lugar en el mercado; bajo la premisa de que si los micronegocios crecen también lo hará Wabi. El objetivo comercial de Wabi en el primer trimestre de 2020 era acompañar e impulsar la integración de 800 mil "tienditas de la esquina" al comercio en línea (Forbes, 2020).

$\mathrm{Su}$ funcionamiento es sencillo, el cliente solicita los productos por medio de una aplicación disponible para IOS y Android, la plataforma identifica al negocio más cercano y envía la solicitud, el pedido llega a la tienda y si lo acepta, un repartidor del negocio es quien entrega los productos de manera directa.

Para los comercios, Wabi tiene disponible un sitio para registrarse, la plataforma sirve de enlace con proveedores de consumo masivo y mayoristas a quienes pueden comprar productos para venderlos a sus clientes a través de la app o de manera directa a sus clientes en la tienda.

Debe considerarse que estos micronegocios dependen de los vecinos de su negocio y de transeúntes que pasan frente al negocio para poder vender, y estos últimos deciden hacer compra de productos de último momento o no programada. Justo esta característica o comportamiento de los clientes hizo que, durante la pandemia, los niveles de venta de estas tiendas se vieran muy mermados debido a una reducción del tránsito de personas.

\section{MÉTODO}

Para alcanzar el objetivo de la investigación se llevó a cabo una investigación de corte cualitativo; se aplicaron entrevistas 
semiestructuradas a micronegocios (tendajones, misceláneas, tiendas de abarrotes) durante el mes de mayo de 2021 en la zona sur de la ciudad de Puebla. Las variables del estudio se muestran en la Tabla 1.

Tabla 1. Variables del estudio.

Variables

Características básicas del micronegocio

Impulsores para ingresar al comercio electrónico.

Factores que le impulsaron a integrarse al comercio electrónico

Beneficios. Reales y percibidos, antes y después de haber ingresado en las plataformas participantes

Adecuaciones realizadas para poder ingresar a las plataformas elegidas

Problemas

Detectados por el uso de la plataforma o app elegida

Incentivos para continuar participando en el comercio electrónico

Fuente: Elaboración propia.

La validez de contenido de las preguntas dentro de la entrevista estructurada, se llevó a cabo a través de la técnica de jueces, quienes revisaron la pertinencia de las variables y sus dimensiones, así como de las preguntas integradas en el instrumento.

La entrevista aplicada constaba de 13 ítems que sondeaban las dimensiones presentadas en la Tabla 1. Las preguntas eran abiertas y el entrevistado podía responder de manera libre sobre los temas sondeados, al ser semiestructurada, se permitía añadir preguntas adicionales o complementarias que permitieran detectar mejor la percepción del entrevistado.

La selección de micronegocios a integrar a la investigación se realizó por un muestreo no probabilístico por conveniencia. El criterio empleado era la cercanía del establecimiento al centro de investigación. En esta etapa de pilotaje, se entrevistó a 3 establecimientos, cada uno perteneciente a una de las categorías de micronegocios a sondear.
Dimensiones

Tipo de micronegocio Número de empleados

Relación de los empleados con el emprendedor Razones

Plataformas (criterios de elección y tiempo de haber ingresado)

Experiencia inicial con cada plataforma Tipo de beneficios

Nivel de satisfacción Productos

Servicios

Otros

Disponibilidad de información

Pagos

Otros

Plataforma actual

Nuevas plataformas

Los resultados de las entrevistas fueron analizados mediante la técnica de análisis de contenidos, para ello se apoyó en Atlas. ti 8 tanto para la generación de las nubes de palabras como en la determinación de relaciones entre variables.

\section{RESULTADOS}

Con los resultados obtenidos de las entrevistas estructuradas se generó la nube de palabras, que se muestra en la Figura 3.

Figura 3. Nube de datos

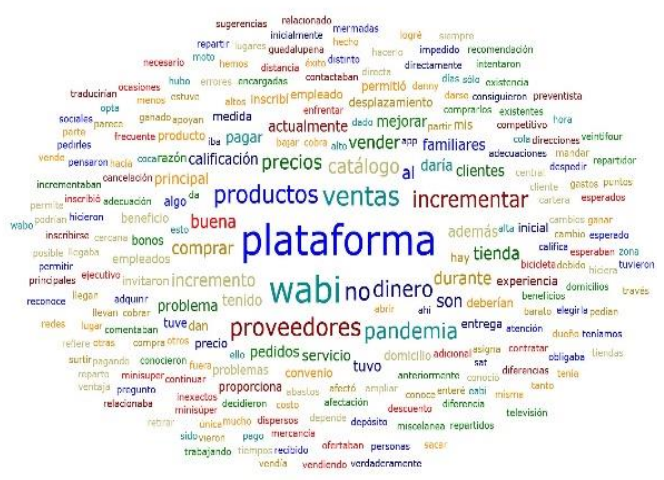

Fuente: Elaboración propia.

Dentro de la nube obtenida en Atlas.ti, se puede identificar que las palabras que más se 
repiten son: plataforma, productos, proveedores, ventas, pandemia, precios, catálogo. Este grupo de palabras son las que concentran la esencia de lo que los microempresarios de este tipo de negocios comentan alrededor de esta plataforma.

La Figura 4, establece las relaciones entre las variables analizadas dentro del instrumento empleado.

Figura 4. Relaciones entre variables

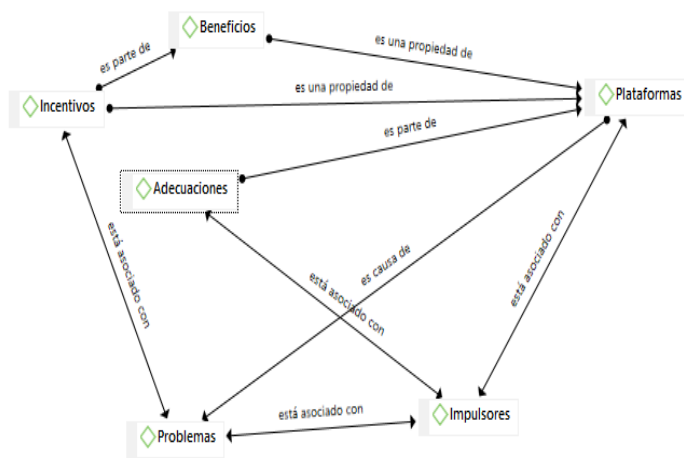

Fuente: Elaboración propia

La codificación resultante de las variables y sus dimensiones, permiten reconocer que para el microempresario los impulsores son percibidos de la misma manera que los beneficios esperados, por lo que no parece haber criterios prestablecidos distintos a estos beneficios, que apoyen la decisión de ingreso. Adicionalmente se reconoce que no existe conocimiento de otro tipo de plataforma que pudiera servirles para poder incrementar sus ventas o aumentar el campo de acción del micronegocio. Algunas frases para destacar dentro de las entrevistas son: Me inscribí en Wabi para incrementar el desplazamiento de mis productos. Al inscribirme a Wabi esperaba incrementar las ventas.

Lo anterior era de esperarse dado que no había una experiencia previa con el comercio electrónico, en la mayoría de los casos, al ser esta su primer contacto dentro de este ámbito va a constituir un importante decisor para repetir la experiencia en otras plataformas existentes o emergentes.

En relación con la brecha (gap) entre los beneficios esperados y los recibidos, se pudo constatar que esta variable desde la dimensión de tipos de beneficios era condicionante de la decisión de continuar trabajando con esta u otras plataformas, otros factores relacionados fueron el nivel de satisfacción, la percepción de problemas y los incentivos recibidos. Los beneficios esperados coincidían en cuanto a incremento en el nivel de ventas y desplazamiento de productos; algunos microempresarios manifestaron haber logrado parcialmente sus objetivos iniciales o haber conseguido un beneficio que no esperaban, como es el caso de ampliar la cartera de clientes.

Como resultado de esta brecha entre los beneficios esperados y los recibidos, el microempresario se calificaba como más o menos satisfecho con la app y su uso. En este punto se logró cuantificar el nivel de satisfacción el que, en promedio, fue 8.3; aunque algunos entrevistados indicaron que la calificación podría variar dependiendo de los aspectos a evaluar.

De manera general todos los microempresarios coincidieron en que hubo necesidad de hacer alguna adecuación para poder ingresar y mantenerse en la plataforma. Las principales adecuaciones mencionadas iban desde incremento de la variedad de productos, acorde al catálogo que manejaba la plataforma; darse de alta ante el SAT (Sistema de Administración Tributaria); adquirir equipo de transporte para atender los pedidos e incluso contratar a personal para poder repartir. La siguiente frase se toma de la entrevista de uno de los microempresarios: Para poder vender en wabi tuve que comprar inicialmente una bicicleta, pero a medida que los pedidos incrementaban, llegaba a tener hasta 10 pedidos en una hora y en lugares dispersos, me obligaba a tener que mandar al repartidos a un lugar mientras yo (dueño) iba a repartir a otro.

De la misma manera, sobre la variable de problemas detectados al usar la app, de reconoció que, en algunos casos, había errores en el manejo de la información por parte de la app, por ejemplo, había imprecisiones en las direcciones de los clientes, productos solicitados o precios publicados. Lo anterior 
llevaba a que en ocasiones el cliente se quejara de servicio recibido y esto impactara en la calificación al establecimiento y la pérdida de los bonos relacionados a la calificación del cliente. A diferencia de lo esperado, el sistema de pagos es bien percibido por los microempresarios, ya que consideran que les facilitaba adquirir productos con los proveedores de la app e incluso con goce de promociones. Asimismo, el sistema de transferencia a las cuentas bancarias de los negocios es fácil. Una frase para destacar en las entrevistas fue: Entrevistado 1. Un problema que tiene la plataforma es que hay que comprar a los proveedores con los que Wabi tiene convenio y ellos tiene precios más altos, por lo que es más barato ir a comprarlos a la central de abastos. Entrevistado 2. Yo prefiero comprar con los proveedores de la app porque me permite aprovechar las promociones que tienen y además me lo traen a mi negocio.

Y finalmente, en cuanto a los incentivos percibidos para continuar en esta u otra plataforma, se encontró que hay una variedad de opiniones al respecto, ya que algunos microempresarios sí optarían por explorar nuevas plataformas, mientras que otras prefieren ni siquiera continuar trabajando con Wabi, como lo reflejan algunas de las frases mencionadas por los entrevistados: Ya dentro de la plataforma, lo que verdaderamente conseguí fue incrementar la cartera de clientes, ya que además de que los clientes me pedían en wabi, también me contactaban directamente en la tienda para pedirles productos.

\section{CONCLUSIONES}

El presente artículo describe los resultados del pilotaje del instrumento en los micronegocios que habían tenido alguna experiencia con el uso de la plataforma Wabi. De lo anterior, se puede concluir que el instrumento empleado es adecuado, aunque es factible de mejorar en algunos aspectos.

Es posible reconocer la importancia que este tipo de aplicaciones (apps) tienen para potenciar y apoyar a micronegocios como tiendas, misceláneas o tendajones, en su integración a las tendencias de comercio electrónico, ya que le permite llevar a cabo operaciones que, dado el tamaño del negocio, no podrían realizarse con la eficiencia que le proporciona la app.

Cabe reconocer que la variedad de percepciones en los dueños de los micronegocios es amplia, lo mismo se encontró emprendedores con una opinión positiva sobre su experiencia en plataforma $y$ otros que la misma fue negativa.

El instrumento generado permitió medir las variables y sus dimensiones conforme a lo establecido al inicio de la investigación, aunque se acepta que las variables: impulsores, beneficios y problemas, comparten aspectos relacionados, por lo que debe hacerse una precisión de ellos dentro de las preguntas consideradas en el instrumento. Asimismo, se detectó que el uso de instrumento de carácter cuantitativo podría mejorar la percepción y determinación de las razones y experiencia de estos microempresarios con el uso de app como la de Wabi. Por lo que se propone que la dimensión de nivel de satisfacción sea desglosada en varios aspectos para una medición más adecuada.

Los resultados preliminares permiten en primera instancia, asumir que este tipo de aplicaciones ha impacto al menos parcialmente, en el logro de las expectativas iniciales de los microempresarios y les ha permitido mostrarse receptivos a continuar usándolas y a integrarse al comercio electrónico. A través del uso de este tipo de plataformas, los microempresarios podrán fortalecer sus competencias relacionadas con el manejo de TICs y se favorecerá la inclusión financiera y la continuidad en el comercio electrónico. 


\section{REFERENCIAS}

Fontalvo, S.I. (2013). El comercio electrónico como recurso de competitividad para las PYMES de la Sierra Nevada de Santa María. Revista electrónica Gestión de las Personas y Tecnología. 6(17): 60-71. En https://www.redalyc.org/articulo.oa?id=477847109005

Forbes (2020). Concanaco lanza Wabi, la app para que tienditas vendan en línea. En https://www.forbes.com.mx/negocios-concanaco-app-wabi-tienditas-ventas-en-linea/

Instituto Nacional de Estadística, Geografía e Informática (2014). Tiendas de conveniencia en México. INEGI. En https://www.inegi.org.mx/contenidos/programas/ce/2014/doc/minimonografias/tieconv_ ce14.pdf

Instituto Nacional de Estadística, Geografía e Informática (2019). Censos económicos 2019. Características de los establecimientos de propietarios y propietarias. En https://www.inegi.org.mx/contenidos/productos/prod_serv/contenidos/espanol/bvinegi/p roductos/nueva_estruc/889463900900.pdf

Instituto Nacional de Estadística, Geografía e Informática (2020a). Censos económicos 2019. INEGI. https://www.inegi.org.mx/programas/ce/2019/?fbclid=IwAR2yC6Ac9S3dI9YkB1fWRw r8ejRmJk2jtZW47TPAgIJ724rWcbCVfUR1Xy4\#Tabulados

Instituto Nacional de Estadística, Geografía e Informática (2020b). INEGI informa. INEGI. En https://www.facebook.com/INEGIInforma/posts/3948648828512771/

Martínez López, E.A. (2013). El sistema de negocios como instrumento de referencia contextual para la formación de saberes productivos de la microempresa en México. Entreciencias: diálogos en la Sociedad del Conocimiento. 1(2): 173-187 En https://www.redalyc.org/articulo.oa?id=457645124008 